\title{
Soziale Netzwerke, familiales Sozialkapital und kindliche Gesundheit
}

\section{Daniel Lois}

\section{Überblick}

- Soziale Kindernetzwerke bestehen im engsten Kreis vorwiegend aus Mitgliedern der Kernfamilie (Eltern, Geschwister) und im erweiterten Kreis aus weiteren Familienmitgliedern wie Großeltern und Freunden. Netzwerkgröße und Anteil der Freunde im Netzwerk steigen mit dem Alter.

- Ein Literaturüberblick zeigt, dass die kindliche Gesundheit sowohl direkt durch das soziale Netzwerk des Kindes beeinflusst wird als auch indirekt durch das soziale Netzwerk der Eltern.

- Von den verschiedenen theoretischen Mechanismen, die zur Erklärung dieser Befunde infrage kommen - z. B. soziale Unterstützung, soziale Ansteckung oder soziale Kontrolle -, ist der Unterstützungsmechanismus am besten empirisch bestätigt. Allerdings sind „echte“ Netzwerkstudien, in denen Familiennetzwerke namensbasiert aufgespannt werden, im hier betrachteten Altersbereich eher selten.

- Familiales Sozialkapital korreliert in westlichen Industrienationen positiv mit den sozioökonomischen Ressourcen der Eltern. Für Schwellenund Entwicklungsländer zeigt sich, dass die kindliche Gesundheit hier verstärkt vom Vorhandensein sozialer Unterstützung abhängig ist.

Der Autor bedankt sich bei den Herausgebern sowie Holger von der Lippe für ihre Unterstützung bei der Literaturrecherche sowie hilfreichen Anregungen.

D. Lois $(\bowtie)$

Neubiberg, Deutschland

E-Mail: daniel.lois@unibw.de

(C) Der/Die Herausgeber und der/die Autor(en) 2020

A. Klärner et al. (Hrsg.), Soziale Netzwerke und gesundheitliche Ungleichheiten, https://doi.org/10.1007/978-3-658-21659-7_7 


\section{$1 \quad$ Einleitung}

Seitdem sich Ende der 1980er Jahre das sogenannte Salutogenesemodell etabliert hat, suchen Forscher und Praktiker im Feld der Gesundheitsförderung nicht nur nach Faktoren, die krank machen, sondern auch nach Faktoren und dynamischen Wechselwirkungen, die zur Entstehung und Erhaltung von Gesundheit führen. Soziale Beziehungen gelten hierbei als hilfreich und unterstützend. Ihre Verbesserung ist ein Ziel der Gesundheitsförderung.

Der vorliegende Beitrag beschäftigt sich mit der Familie als einen Kernbereich sozialer Beziehungen und nimmt zudem die physische und mentale Gesundheit von Kindern (bis 13 Jahre) in den Blick. Familiale Einflüsse auf die kindliche Gesundheit sind vielfältig (vgl. Rattay et al. 2012): Von klein auf bis ins Jugendalter lernen Kinder in der Familie den Umgang mit ihrem Körper, mit Gesundheit, Krankheit sowie entsprechenden Bewältigungsstrategien. In der alltäglichen Interaktion in der Familie und durch die sozialen Kontexte, zu denen die Familie einen Zugang ermöglicht, erwerben Kinder und Jugendliche grundlegende Kenntnisse und Einstellungen, die zu ihrer Entwicklung in psychischer, körperlicher, sozialer und kognitiver Hinsicht beitragen. Die gesundheitsbezogene Sozialisation erfolgt

\footnotetext{
„insbesondere über familiale Alltagspraktiken wie die Gestaltung der Mahlzeiten, das Bewegungs- und Freizeitverhalten in der Familie, feste Tageszeiten für das Aufstehen und Ins-Bett-Gehen, die Inanspruchnahme von medizinischen Leistungen sowie über familiale Haltungen zum Beispiel zur Hygiene. Aber auch gesundheitsabträglichen Praktiken wie dem Konsum von Tabak und Alkohol sind Heranwachsende in ihrer Familie mehr oder weniger direkt ausgesetzt" (Rattay et al. 2012, S. 146).
}

In der Forschung wird zwischen protektiven Faktoren von Familie und Risikofaktoren unterschieden. $\mathrm{Zu}$ den Risikofaktoren zählen z. B. ungünstige materielle Bedingungen, niedrige Schulbildung der Eltern, Arbeitslosigkeit, beengte Wohnverhältnisse, familiale Konflikte, körperliche oder mentale Erkrankungen eines Elternteils, schlechte Verfügbarkeit primärer Bezugspersonen im Kleinkindalter, Vernachlässigung oder Gewalt (Tiber Egle et al. 2002).

Beim vorliegenden Beitrag handelt es sich um einen Literaturüberblick, der sich vorwiegend mit protektiven sozialen Faktoren beschäftigt, welche die kindliche Gesundheit beeinflussen. Im Mittelpunkt stehen dabei a) direkte Einflüsse des kindlichen sozialen Netzwerkes, z. B. die soziale Unterstützung durch die Eltern und b) indirekte Einflüsse des elterlichen Netzwerks auf das Kind, z. B. in Form von emotionaler oder instrumenteller Unterstützung der Eltern durch die Großeltern. 
Zunächst wird in Abschn. 2 besprochen, wie soziale Netzwerke von Kindern zusammengesetzt sind, welche Funktionen sie theoretisch erfüllen und welche Effekte auf die Gesundheit daraus resultieren könnten. In die Diskussion integriert wird hier der Begriff des ,family social capital“, der auf James S. Coleman zurückgeht. In Abschn. 3 wird dann als Ausgangspunkt kurz auf Studien eingegangen, die den sozioökonomischen Status der Familie als Prädiktor für den Gesundheitszustand des Kindes verwenden. Davon ausgehend wird in Abschn. 4 im Rahmen eines Literaturüberblicks auf Netzwerkeffekte eingegangen, die zudem in Abschn. 5 noch einmal speziell dahin gehend überprüft werden, inwiefern sie unabhängig von klassischen Ungleichheitsdimensionen auftreten bzw. wie sie mit diesen Dimensionen verknüpft sind. In Abschn. 6 erfolgt eine abschließende Diskussion der Befunde.

\section{Kindernetzwerke: Struktur, Funktionen und Effekte auf die Gesundheit sowie familiales Sozialkapital}

\subsection{Strukturen, Funktionen und Gesundheitseffekte von sozialen Netzwerken von Kindern}

Nestmann und Wehner (2008, S. 22-27) beschreiben, aufbauend auf Vaux (1988), in welchem Spektrum soziale Netzwerke die Gesundheit von Kindern beeinflussen können. Sie nehmen dabei eine entwicklungspsychologische Perspektive ein. Beim Kleinkind ermöglichen hiernach ein sicherer Halt und eine vertrauensvolle Bindung zu den engsten Bezugspersonen nach der Bindungstheorie (Bowlby 1975) eine Exploration neuer sozialer Beziehungen und Lebenssphären. Über die Mutter-Kind-Beziehung hinaus sollte jedoch schon früh ein Augenmerk auf eine mögliche Netzwerkperspektive gelegt werden, die neben anderen Kontaktpersonen der Kleinkinder (z. B. Väter, Geschwister, Großeltern, Pflegepersonen) auch die Einflüsse sogenannter Exosysteme (Bronfenbrenner 1981) aufgreift. Hiermit ist gemeint, dass Personen und Institutionen Kinder, vermittelt über ihre engsten Bezugspersonen, indirekt tangieren. Neben den sozialen Netzwerken der Kinder selbst sind demzufolge auch die Netzwerke der Eltern in die Analyse mit einzubeziehen (vgl. auch Cochran und Brassard 1979).

Im direkten Kontakt zu Netzwerkmitgliedern der Eltern erhalten die Kinder verschiedene kognitive und soziale Anregungen, die ihre Entwicklung stimulieren: andere Interaktionsstile und Zuwendungsformen, divergierende Interaktionsinhalte (z. B. Reden, Spielen, Vorlesen) und andere Interaktionssettings 
(z. B. der Haushalt der Großeltern). Neben diesen Anregungen erfahren die Kinder andere Formen der Unterstützung und Regulation. Mitglieder des elterlichen Netzwerks dienen zudem als Verhaltensmodelle (soziales Lernen) und bieten den Kindern Interaktionsgelegenheiten, die ihre Sozialkompetenz stärken.

Personen aus dem Netzwerk der Eltern wie Großeltern, Freunde oder Nachbarn können die Kinder auf verschiedene Weise indirekt beeinflussen: Sie geben den Eltern explizite oder implizite Rückmeldungen zur Kinderversorgung und Erziehung, unterstützen sie bei Erziehung und Versorgung (z. B. bei Notfällen und Krankheit), bestätigen die Eltern emotional in ihrer Elternrolle und geben Rückhalt. Zudem stellen Netzwerkpersonen Lernmodelle dar, die den Zugang zu einer größeren Bandbreite von Interaktionsstilen, Strategien der Alltagsführung oder Konfliktlösungskompetenzen bieten. Neben den aufgeführten positiven Impulsen sind jedoch entsprechend auch negative Einflüsse wie z. B. ungebetene Ratschläge, widersprüchliche Vorgaben oder negative Verhaltensbeispiele zu berücksichtigen.

Hieraus lassen sich bereits einige zentrale Mechanismen ableiten, durch die soziale Netzwerke des Kindes selbst oder elterliche Netzwerke die kindliche Gesundheit beeinflussen (zu theoretischen Mechanismen siehe Kap. „Soziale Beziehungen, soziales Kapital und soziale Netzwerke“ sowie Kap. „Wirkmechanismen in sozialen Netzwerken"). Heuristisch sind vier Aspekte differenzierbar:

- Soziale Unterstützung: Im Hinblick auf soziale Unterstützung unterscheiden Erhart und Ravens-Sieberer (2008) zwischen einer Abschirm-, Puffer- und Toleranzwirkung. Emotionale oder instrumentelle Unterstützung beugt Krisensituationen vor (1., Abschirmwirkung), kann in eingetretenen Krisensituationen negative Auswirkungen durch eine produktive Verarbeitung der Anforderungen reduzieren (2., Pufferwirkung) und stärkt Fähigkeiten, mit bereits eingetretenen Gesundheitsstörungen umzugehen (3., Toleranzwirkung). Beispiele für die Abschirmwirkung wären das warme Anziehen des Kindes im Winter oder eine vitaminreiche Ernährung. Stärken Netzwerkpersonen das Selbstwertgefühl und den Bewältigungsoptimismus in Stresssituationen, z. B. bei Schulstress des Kindes, wäre dies ein Beispiel für die Pufferwirkung. Wird ein krankes Kind von Netzwerkpersonen emotional unterstützt, zeigt sich hierin beispielhaft die Toleranzwirkung.

- Soziale Kontrolle: Eltern und auch Personen aus dem Netzwerk der Eltern (z. B. Großeltern) sind in Familien mit hoher Kohäsion durch (informelle) soziale Kontrolle eher in der Lage, Kinder z. B. vor „risk-fashion“ Aktivitäten 
oder gesundheitsgefährdenden Verhaltensweisen (etwa Drogenkonsum) fernzuhalten. Bei hoher Kohäsion kann der Gesundheitszustand des Kindes zudem besser überwacht und ggf. darauf reagiert werden (,monitoring“).

- Soziale Beeinflussung: Eltern haben in Familien mit hoher Kohäsion eher die Möglichkeit, beispielsweise das Essverhalten der Kinder im Rahmen von regelmäßigen gemeinsamen Mahlzeiten aktiv zu beeinflussen und auch das Wissen zu gesunder Ernährung zu fördern. Gleiches gilt für physische Aktivität (z. B. Sport) oder Medienkonsum. Enge familiale Sozialbeziehungen fördern außerdem soziale Gemeinschaft und eine positive Gemütslage. Dies vermeidet negative Isolationseffekte wie Depression oder Vernachlässigung von Ernährung oder Selbstpflege.

- Soziale Ansteckung: Die Eltern selbst, aber auch Personen aus dem sozialen Netzwerk der Eltern, stellen erstens für Kinder und Jugendliche Verhaltensmodelle dar, deren Einstellungen und Verhaltensweisen im Rahmen von sozialem Lernen (Bandura 1977) übernommen werden können. Zweitens besteht ein indirekter Mechanismus darin, dass Betreuungspersonen des Kindes selektiv Beziehungen zu Netzwerkpersonen aufbauen bzw. erhalten, die ähnliche Einstellungen und Verhaltensweisen im Bereich der Gesundheit aufweisen wie sie selbst (Homophilie; Kennedy-Hendricks et al. 2015).

Im nächsten Schritt stellt sich die Frage, welche Personen aus dem Netzwerk des Kindes bzw. aus dem elterlichen Netzwerk welche Funktionen übernehmen und ob es hier je nach Alter des Kindes Unterschiede gibt. Hinsichtlich der Struktur kindlicher Netzwerke verwenden Levitt et al. (1993) - in Anlehnung an Kahn und Antonucci (1980) - die Metapher der ziehenden Karawane, einer Idealtypik der strukturellen Netzwerkentwicklung über den Lebensverlauf. Hiernach durchzieht zunächst ein kleiner Konvoi der engsten Familie die frühe Kindheit, reichert sich anschließend schnell durch Verwandtschaft, Freundschaft und Nachbarschaft an und vergrößert sich in der späten Kindheit bzw. im Jugendalter, in denen die Peers und erste institutionelle Kontakte (Kindergarten, Schule) hinzutreten. Aufbauend auf diesem „,convoy model“ verwenden Levitt et al. (1993) konzentrische Kreisdiagramme, um Netzwerkpersonen der Kinder (hier zwischen 7 und 14 Jahre) voneinander abzugrenzen, die den innersten Zirkel des Netzwerks (größte Wichtigkeit für das Kind und Nähe zum Kind) oder den mittleren und äußeren Zirkel bilden. Es zeigt sich, dass der innerste Zirkel fast ausschließlich aus engen Familienmitgliedern (Eltern, Geschwister) bzw. anderen Familienmitgliedern wie Großeltern besteht. Der mittlere Zirkel ist heterogen zusammengesetzt (es überwiegen Familienmitglieder außerhalb der Kernfamilie aber auch Freunde 
gewinnen an Bedeutung) und im äußeren Zirkel stellen Freunde die größte Gruppe dar. Mit dem Alter der Kinder steigen die Netzwerkgröße, der Anteil der Freunde und das wahrgenommene Unterstützungspotenzial im Netzwerk (insbesondere durch Freunde; siehe auch Bost et al. 2004).

Boosman et al. (2002) zeigen ferner in einer differenzierten Studie zu sozialen Netzwerken von Kindern im Alter von 4-8 Jahren, welche Arten von Netzwerkeinflüssen (soziale Kontrolle, soziale Unterstützung und Kinderbetreuung) von welchen Netzwerkpersonen ausgehen. Dabei zeigt sich erwartungsgemäß, dass alle drei Funktionen allgemein am häufigsten von den Eltern erfüllt werden. Erfolgt jedoch eine Unterteilung nach Unterstützungsarten, zeigt sich ein differenzierteres Bild: Unterstützung, die sich in Form von Geselligkeit und Spielgelegenheiten äußert, wird am häufigsten von Peers zur Verfügung gestellt. Auch Konflikte werden mit Peers häufiger ausgetragen als mit den Eltern. Großeltern ähneln strukturell den Eltern - allerdings auf niedrigerem Niveau. Sie üben nach den Eltern relativ häufig soziale Kontrolle aus, sind relativ stark für die Kinderbetreuung (z. B. Essen, Anziehen) verantwortlich und bieten auch emotionale wie instrumentelle Unterstützung. Geschwister spielen nach den Eltern und den Peers ebenfalls eine wichtige Rolle im Bereich der Geselligkeit, das Konfliktniveau mit Geschwistern ist jedoch niedriger ausgeprägt als bei Peers. Lehrer werden etwas überraschend selten als signifikante Einflusspersonen benannt, sogar im Bereich „informational support“. Schließlich zeigt sich, dass auch Kuscheltiere und Puppen von den Kindern stellenweise als symbolische Netzwerkpersonen eingeordnet werden, z. B. im Bereich der emotionalen Unterstützung.

Nur selten wird in der Literatur diskutiert, welche Eigenschaften einzelne Ties bzw. das Netzwerk insgesamt aufweisen müssen, damit die oben diskutierten Mechanismen wirken. Erhart und Ravens-Sieberer (2008) deuten an, dass die Abschirm-, Puffer- und Toleranzwirkung sozialer Unterstützung vor allem dann zum Tragen kommt, wenn zum einen mehrere Unterstützer zur Verfügung stehen und Unterstützung zum anderen langfristig angelegt ist. In ähnlicher Weise erwarten Kana'iaupuni et al. (2005) in einer mexikanischen Studie, dass soziale Netzwerke, die entweder einen hohen Anteil von Blutsverwandten (Eltern und Geschwister) oder einen hohen Anteil von ,extended kin“ enthalten, mehr soziale Unterstützung bzw. eine besonders intensive Form der Unterstützung bereitstellen können. Unter „,extended kin“ werden dabei Vertrauenspersonen (,persons de confianza") in einem kulturell verankerten reziproken Unterstützungssystem sowie Paten der Kinder („Compadrazgo“) subsumiert. Zusätzlich wird erwartet, dass sich räumliche Nähe, Koresidenz und eine hohe Kontakthäufigkeit positiv 
auf das Unterstützungspotenzial auswirken. Somit kann vorsichtig vermutet werden, dass dichte Netzwerke mit hohem Verwandtenanteil, die entsprechend vorwiegend aus Strong Ties bestehen, besonders förderlich für die kindliche Gesundheit sein sollten.

\subsection{Der Ansatz des familialen Sozialkapitals von Kindern}

Anschlussfähig an die besprochene Netzwerkperspektive - zugleich aber breiter ist ferner der auf Coleman (1990) zurückgehende Begriff des ,family social capital". Hier wird postuliert, dass der familiäre Hintergrund eines Kindes aus drei Komponenten besteht:

- finanzielles Kapital (die finanziellen Ressourcen, die für den Haushalt insgesamt und das Kind zur Verfügung stehen),

- elterliches Humankapital (z. B. die kognitiven Fähigkeiten und Bildungsabschlüsse der Eltern),

- Sozialkapital (die Ressourcen, die im Rahmen von sozialen Beziehungen zur Verfügung stehen und für die kognitive und soziale Entwicklung von Kindern und Jugendlichen nützlich sind).

Das Sozialkapital stellt allgemein eine konzeptionelle Verbindung her zwischen den Eigenschaften individueller Akteure und ihren unmittelbaren sozialen Kontexten in Haushalt, Schule und Nachbarschaft - mithin also auch außerfamiliale Netzwerkbeziehungen. Das intra-familiale Sozialkapital bezieht sich auf die Eltern-Kind-Beziehung und manifestiert sich durch die Zeit und die Aufmerksamkeit, die Eltern aufbringen, indem sie mit ihren Kindern interagieren, soziale Kontrolle ausüben und ihr Wohlbefinden fördern. Operationalisiert wird familiales Sozialkapital in der Forschung über Indikatoren, die sich zwei Hauptdimensionen zuordnen lassen:

- die strukturelle Dimension (familiale Struktur): z. B. Kernfamilie (mit zwei biologischen Elternteilen im Haushalt) vs. Stieffamilie oder alleinerziehende Eltern; Anzahl der Geschwister oder

- die funktionale Dimension (das Vorhandensein förderlicher Interaktionen zwischen Eltern und Kindern). 
Tab. 1 Konzepte zur Messung von familialem Sozialkapital

\begin{tabular}{|c|c|c|}
\hline Konstrukt & Unterkonstrukt & Beispielitems \\
\hline \multirow[t]{4}{*}{ Familiale Kohäsion } & Kollektive Wirksamkeit & $\begin{array}{l}\text { Wahrnehmung, als Familie gut zu } \\
\text { funktionieren }\end{array}$ \\
\hline & Informelle Kontrolle & $\begin{array}{l}\text { Wie viele Stunden sind Kinder nach der } \\
\text { Schule ohne Eltern zu Hause? } \\
\text { Kennen Eltern Freunde des Kindes? } \\
\text { Erlauben Eltern dem Kind, mit ihnen } \\
\text { unbekannten Freunden auszugehen? } \\
\text { Überprüfen Eltern, ob Kinder ihre } \\
\text { Hausaufgaben erledigt haben? }\end{array}$ \\
\hline & Soziale Interaktion & $\begin{array}{l}\text { Häufigkeit gemeinsamer Eltern-Kind- } \\
\text { Aktivitäten wie z. B. Mahlzeiten, } \\
\text { Spiele, Gespräche, Feiern }\end{array}$ \\
\hline & $\begin{array}{l}\text { Gefühl der } \\
\text { Zugehörigkeit }\end{array}$ & $\begin{array}{l}\text { Respektieren sich Familienmitglieder } \\
\text { gegenseitig? } \\
\text { Empfinden sie gegenseitig Loyalität und } \\
\text { Vertrauen? }\end{array}$ \\
\hline \multirow[t]{3}{*}{$\begin{array}{l}\text { Familiale } \\
\text { Unterstützung }\end{array}$} & $\begin{array}{l}\text { Emotionale } \\
\text { Unterstützung }\end{array}$ & $\begin{array}{l}\text { Sprechen Familienmitglieder über } \\
\text { Sorgen? } \\
\text { Sind Verwandte verlässliche Personen, } \\
\text { die bei ernsten Problem helfen? } \\
\text { Wird Familienmitgliedern emotionale } \\
\text { Unterstützung, Empathie und Liebe } \\
\text { entgegengebracht? }\end{array}$ \\
\hline & $\begin{array}{l}\text { Instrumentelle } \\
\text { Unterstützung }\end{array}$ & Eltern helfen bei den Hausaufgaben \\
\hline & Konflikt & $\begin{array}{l}\text { Wie häufig kritisieren sich } \\
\text { Familienmitglieder? } \\
\text { Konfligieren persönliche Ziele mit } \\
\text { denen der Familie? }\end{array}$ \\
\hline \multirow[t]{2}{*}{$\begin{array}{l}\text { Soziales Netzwerk } \\
\text { (des Kindes bzw. der } \\
\text { Eltern) }\end{array}$} & Netzwerkstruktur & $\begin{array}{l}\text { Netzwerkgröße, -dichte und -zentralität; } \\
\text { Geschlechts- und Alterskomposition; } \\
\text { Reichweite des Familiennetzwerks } \\
\text { (Eltern, Geschwister, Schwiegereltern, } \\
\text { weitere Verwandte) }\end{array}$ \\
\hline & Beziehungsqualität & $\begin{array}{l}\text { Wohnortnähe und Kontakthäufigkeit, } \\
\text { emotionale Nähe }\end{array}$ \\
\hline
\end{tabular}

Quelle: Eigene Darstellung basierend auf Alvarez et al. 2017, S. 19 f 
Der Begriff des „family social capital“ leidet zum Teil unter seiner theoretischen Unschärfe und der Breite der Messindikatoren, die hier subsumiert werden. ${ }^{1}$ Dem Sozialkapital zugerechnet werden sowohl die familiale Kohäsion, die Qualität der Eltern-Kind-Beziehung (z. B. Kontakthäufigkeit, emotionale Nähe) als auch strukturelle Aspekte des kindlichen Netzwerks bzw. des Elternnetzwerks. Zu letzteren zählen z. B. Netzwerkgröße, -dichte und -zentralität (Alvarez et al. 2017).

Alvarez et al. (2017) haben hierzu auf Basis eines Literaturüberblicks eine Übersicht erstellt, die in Tab. 1 - etwas verkürzt - wiedergegeben wird. Die Bereiche der familialen Kohäsion und der familialen Unterstützung lassen sich als Ausdifferenzierungen der funktionalen Dimension nach Coleman verstehen und das Konstrukt des familialen Netzwerks als Repräsentanz der strukturellen Dimension.

$\mathrm{Zu}$ ergänzen bleibt, dass der Begriff der sozialen Kohäsion voraussetzungsvoller ist als der Begriff des Sozialkapitals, und vor allem in der Familienpsychologie als zentrales Kriterium familialer Funktionsfähigkeit beschrieben wird (z. B. Dilworth-Anderson et al. 2005). So ist es möglich, dass trotz eines ausgeprägten Sozialkapitals keine soziale Kohäsion besteht. Umgekehrt erscheint die Abwesenheit von Sozialkapital bei gegebener sozialer Kohäsion nur schwer denkbar.

\section{$3 \quad$ Soziale Ungleichheit und kindliche Gesundheit}

Eine umfangreiche Literatur widmet sich dem Zusammenhang zwischen sozialer Ungleichheit und (kindlicher) Gesundheit. Zugrunde liegt hier das Konzept des sozioökonomischen Status, d. h. die individuelle Position in einem durch

\footnotetext{
${ }^{1}$ Das Konzept des familialen Sozialkapitals von Coleman (1990) sowie die Operationalisierung des Konzepts in der Forschung sind in mehrfacher Hinsicht kritisiert worden (Alvarez et al. 2017; Morrow 1999). Es wird eingewendet, dass insbesondere Kinder und Jugendliche lediglich als Nutznießer des Sozialkapitals gesehen werden und dabei übersehen wird, wie sie selbst zu dessen Entstehung beitragen (Morrow 1999). Kritisch ist zudem anzumerken, dass der Begriff des ,,family) social capital“ durch die Vielzahl der in ihm vereinigten Konzepte unscharf wird. Er deckt sowohl strukturelle Aspekte ab (z. B. die Familienform) als auch konkrete Unterstützungsleistungen aus dem Netzwerk bis hin zu Zugehörigkeitsgefühlen, Interaktionsmustern oder der emotionalen Nähe zwischen Eltern und Kindern (Morrow 1999). Hier fehlt die konzeptionelle Abgrenzung zu anderen, zum Teil trennschärferen Begriffen wie z. B. dem Lebensstil (Wippermann 2009).
} 
Ungleichheit in der Verteilung von Privilegien und Wohlstand geprägten Gesellschaftsgefüge. Damit gehen Vor- und Nachteile bezüglich der Verfügbarkeit von verschiedenen Gütern einher. Zu diesen zählen zum einen klassische Güter wie Einkommen, Vermögen, Macht, Sozialprestige, Bildung oder Wissen und zum anderen - wie etwa im Konzept der sozialen Lage (Hradil 1987) - auch die soziokulturelle Teilhabe in den Lebensbereichen Arbeit, Ausbildung, Wohnen, Freizeit und Kultur.

Lampert und Schenk (2004) systematisieren in einem Mehrebenenmodell verschiedene theoretische Erklärungsansätze zum Zusammenhang zwischen sozialer Ungleichheit und kindlicher Gesundheit. Hiernach steht die soziale Lage des Haushaltes, gemessen z. B. über Schichtzugehörigkeit, Einkommenssituation und Familienform (Alleinerziehende) in einer Beziehung zu Lebensbedingungen und Teilhabechancen. Hierzu zählen etwa die materielle Versorgung, die Familiensituation, Wohnverhältnisse, das Freizeitverhalten und die Zusammensetzung der Peers sowie die gewählte Schule bzw. Kindertagesstätte. Lebensbedingungen und Teilhabechancen wirken sich wiederum zum einen auf das Gesundheitsverhalten aus (z. B. Ernährung, körperliche Aktivität, Alkohol- und Tabakkonsum, Mundhygiene) und zum anderen auf die Persönlichkeit, die etwa durch Selbstwertgefühl, Kontrollüberzeugungen, Optimismus oder Aggressivität zum Ausdruck kommt. Gesundheitsverhalten und Persönlichkeit, die sich auch wechselseitig bedingen, führen schließlich zu gesundheitlichen Ungleichheiten, die sich u. a. in Krankheiten, Behinderungen, Unfallverletzungen, subjektiver und psychischer Gesundheit oder Verhaltensauffälligkeiten äußern.

Zahlreiche empirische Studien belegen Zusammenhänge zwischen sozialer und gesundheitlicher Ungleichheit bei Kindern und Jugendlichen. Die entsprechenden Resultate werden hier auf Basis des Forschungsüberblicks von Lampert und Richter (2009), der stellenweise aktualisiert wurde, kurz zusammengefasst:

- Säuglingssterblichkeit: Die bisherige empirische Evidenz, die sich auf regionale Daten beschränkt, zeigt eine erhöhte Säuglingssterblichkeit in unteren sozialen Schichten. Dies gilt auch für Risikofaktoren wie niedrigeres Geburtsgewicht und angeborene Fehlbildungen (Mielck 1998).

- Entwicklungsstörungen: Nach jährlichen Schuleingangsuntersuchungen des Öffentlichen Gesundheitsdienstes (vgl. für das Land Brandenburg: Ellsäßer und Lüdecke 2015) sind Entwicklungsstörungen bei niedrigem Sozialstatus (gemessen über Bildung und Erwerbsstatus der Eltern) deutlich häufiger anzutreffen: Während z. B. Sprach- und Sprechstörungen bei Kindern mit niedrigem Sozialstatus in 43,9\% der Fälle diagnostiziert wurden, waren es bei 
Kindern mit hohem Sozialstatus nur 13,2\%. Ähnlich große Unterschiede zeigen sich z. B. auch bei Wahrnehmungs- und psychomotorischen Störungen, intellektuellen Entwicklungsverzögerungen, emotionalen und sozialen Störungen sowie psychischen Auffälligkeiten. Eine Ursache mag darin liegen, dass die U-Untersuchungen von sozial benachteiligten Bevölkerungsschichten etwas seltener in Anspruch genommen werden (Ellsäßer und Lüdecke 2015)

- Chronische Krankheiten: Chronische Krankheiten bei Kindern weisen ebenfalls einen sozialen Gradienten auf: Nach den Befunden der Brandenburger Einschulungsuntersuchung des Jahres 2015 (Ellsäßer und Lüdecke 2015) sind Kinder mit niedrigem sozialen Status in 23,7\% der Fälle chronisch krank. Beispiele sind somatische Erkrankungen (z. B. Sprach-, Seh- oder Hörstörungen) oder psychische Erkrankungen wie ADHS und emotionale soziale Störungen. Dieser Anteil liegt bei hohem sozialen Status mit 9,5\% deutlich niedriger.

- Unfallverletzungen: Daten zu Unfällen in der Schule, Kita, zu Hause, in der Freizeit und im Straßenverkehr wurden vereinzelt im Hinblick auf Zusammenhänge mit der sozialen Lage untersucht. Eine ältere Studie von Geyer und Peter (1998) zeigt, dass Kinder von un- und angelernten Arbeitern sowie von Facharbeitern häufiger unfallbedingt im Krankenhaus behandelt werden als Kinder von Angestellten und Personen in höheren beruflichen Personen.

- Psychosoziale Gesundheit: Befunde auf Basis der „Health Behaviour in School-aged Children“ (HBSC)-Studie (z. B. Richter et al. 2008) zeigen für Kinder und Jugendliche im Altersbereich 11-15 Jahre, dass die subjektive Gesundheit in Abhängigkeit des familialen Wohlstands besser beurteilt wird. Ähnliche soziale Unterschiede werden auch für psychosomatische Beschwerden wie Kopf-, Bauch- und Rückenschmerzen, Schlafstörungen oder Items zum emotionalen Befinden berichtet.

- Gesundheitsverhalten: Ebenfalls auf Basis der HBSC-Studien lassen sich schließlich statusabhängige Unterschiede z. B. in der Ernährung zeigen (Kinder aus sozial schlechter gestellten Familien essen z. B. seltener frisches Obst und Gemüse) sowie Unterschiede in der Häufigkeit des Tabak- und Alkoholkonsums, die mit dem sozialen Status abnimmt.

Lampert und Richter (2009) resümieren, dass der soziale Status zwar in der Forschungslandschaft sehr unterschiedlich operationalisiert wird (z. B. über die Bildung und die berufliche Stellung der Eltern aber auch über den Wohlstand der Familie), die verschiedenen Statusindikatoren jedoch in ihren Effekten weitgehend übereinstimmen. Sie schließen daraus, dass zwischen der sozialen und gesundheitlichen Lage der Heranwachsenden ein vergleichsweise 
enger Zusammenhang besteht (siehe auch Kap. „Soziale Netzwerke, Gesundheit und gesundheitliche Ungleichheiten im Jugendalter"). Im vorliegenden Beitrag ist nun von besonderem Interesse, inwieweit Netzwerkeffekte auf der einen Seite und Ungleichheitseffekte auf der anderen Seite zusammenhängen. So ist z. B. denkbar, dass die negativen Auswirkungen einer geringen Ausstattung mit sozioökonomischen Ressourcen schwächer ausfallen, wenn die Familie über Kompensatoren in Form von sozialer Unterstützung verfügt.

\section{Soziale Netzwerke, familiales soziales Kapital und kindliche Gesundheit: Ein Literaturüberblick}

Bei der Aufarbeitung des Forschungsstandes wird konzeptuell zwischen zwei Perspektiven unterschieden:

1. Wie wird der Gesundheitszustand der Kinder durch Mitglieder ihres unmittelbaren familialen Netzwerks beeinflusst (Abschn. 4.1)?

2. Welchen Einfluss üben die sozialen Netzwerke der Eltern indirekt auf die Gesundheit der Kinder aus (Abschn. 4.2)?

\subsection{Kindernetzwerke und kindliche Gesundheit}

In diesem Abschnitt liegt das Augenmerk in einem ersten Schritt auf Studien, die Effekte des sozialen Netzwerks des Kindes auf seine Gesundheit in den Blick nehmen und zudem eine Netzwerkmethodologie im engeren Sinne verwenden, d. h. egozentrierte Netzwerke aufspannen. Dieses Kriterium erfüllen die beiden entwicklungspsychologischen Studien von Levitt et al. (1993) sowie Levitt et al. (2005). Zweitens wird der Forschungsstand für Studien kurz zusammengefasst, die den breiteren Begriff des ,family social capital“ verwenden (Bala-Brusilow 2010; Berntsson et al. 2007; Erhart und Ravens-Sieberer 2008; Eriksson et al. 2012; Klocke 2004; Klocke und Lipsmeier 2008; Lau und Li 2011; Morgan und Haglund 2009; Rattay et al. 2012; Wu et al. 2010). Letztere werden größtenteils auch in einem aktuellen Literaturüberblick von Alvarez et al. (2017) besprochen.

Levitt et al. (1993) überprüfen auf Basis einer Stichprobe von N=333 amerikanischen Schulkindern im Altersbereich von 7-14 Jahren, wie affektiv-emotionale und instrumentelle soziale Unterstützung insgesamt und insbesondere soziale Unterstützung aus dem innersten Zirkel des kindlichen Netzwerks (,,convoy model“", siehe Abschn. 2.1) zwei Aspekte der psychischen Gesundheit 
beeinflusst: das positive Selbstkonzept und einen Index zur allgemeinen Gemütslage und Geselligkeit des Kindes. Soziale Unterstützung aus dem sozialen Netzwerk insgesamt, insbesondere aber aus dem innersten Zirkel, der überwiegend aus Mitgliedern der Kernfamilie (Eltern, Geschwister) besteht, üben mittelstarke positive Effekte auf beide Gesundheitsfaktoren aus.

In einer aktuelleren Studie (ebenfalls mit Schulkindern im Altersbereich 9-13 Jahre) erweitern Levitt et al. (2005) diese Befunde um einen typologischen Ansatz. Eine Clusteranalyse führt zu dem Ergebnis, dass es drei Strukturtypen von Kindernetzwerken zu geben scheint: Einen Typ, in dem soziale Unterstützung sowohl von Mitgliedern der Kernfamilie als auch von engen Freunden ausgeht (,close family/friend“), einen Typ mit Unterstützung ausschließlich durch die Familie (,close family“) und einen Typ mit vielfältiger Unterstützung seitens der Kernfamilie, der erweiterten Familie und auch tendenziell von Freunden (,close/extended family“). Wiederum werden mit dem positiven Selbstkonzept, Einsamkeit sowie internalisierten wie externalisierten Verhaltensproblemen (z. B. Gefühl der Wertlosigkeit, körperliche Gewalt) Aspekte des ,psychological adjustment" untersucht. Die Befunde zeigen, dass das Selbstkonzept der Kinder am positivsten, und die Einsamkeit am geringsten ausgeprägt ist, wenn ihre sozialen Netzwerke Unterstützung von mehreren Seiten zur Verfügung stellen, d. h. entweder sowohl von engen Familienmitgliedern und Freunden oder von Mitgliedern der engen und erweiterten Familie (z. B. Onkel, Tanten, Cousinen).

In den Studien, die sich unter dem Begriff des „family social capital“ subsumieren lassen, wird familiales Sozialkapital nicht - wie in den zuvor genannten Studien - über namensbasierte Netzwerkindikatoren, sondern über sogenannte Globalindikatoren erfasst. Hierzu zwei Beispiele: Erhart und Ravens-Sieberer (2008) decken die strukturelle Dimension nach Coleman durch die Familienform (Kernfamilie, Stieffamilie, alleinerziehende Eltern) ab und die funktionale Dimension über a) emotionale Unterstützung durch (Stief-)Eltern und ältere Geschwister und b) instrumentelle Unterstützung durch Eltern in der Schule. Morgan und Haglund (2009) operationalisieren familiales Sozialkapital - dem Kohäsions-Ansatz folgend - zum einen über gemeinsame Aktivitäten in der Familie, z. B. zusammensitzen und unterhalten oder besuchen von Freunden, und zum anderen über das Ausmaß sozialer Kontrolle durch die Eltern („How often does your mother or father try to control everything you do?").

Bei den verwendeten Gesundheitsindikatoren zeigt sich ein breites Spektrum. Abgedeckt werden psychische und psychosomatische Aspekte (z. B. Lebenszufriedenheit, schulischer Leistungsdruck, nervöse Magenprobleme), gesundheitsrelevantes Verhalten (z. B. physische Aktivität, Zähne putzen, gemeinsame Mahlzeiten in der Familie, TV-Konsum, Obst- und Gemüseverzehr, Tabak- und 
Alkoholkonsum), physische Gesundheit (Verletzungen, Übergewicht) und Globalindikatoren zur allgemeinen Gesundheit.

Methodisch werden die Effekte der Sozialkapitalindikatoren auf die Gesundheitsindikatoren in der Regel im Rahmen von hierarchischen Regressionsmodellen bestimmt, die ohne und mit Kontrolle von sozioökonomischen Variablen wie Bildung, Berufsprestige und Einkommen der Eltern geschätzt werden. Die Ergebnisse dieser Analysen führen zu dem Schluss, dass der Sozialkapitalansatz allgemein sehr viel Potenzial zur Erklärung der kindlichen Gesundheit hat. Es zeigt sich sehr konsistent, dass fast alle Sozialkapitalindikatoren die Gesundheitsindikatoren in der erwarteten Weise positiv beeinflussen und dies meist auch bei Kontrolle des sozioökonomischen Status.

Zwei Studien seien besonders hervorgehoben. Die Arbeit von Rattay et al. (2012) ist insofern aufschlussreich, da hier - ermöglicht durch eine relativ große Fallzahl getestet wird, ob die Effekte der Sozialkapitalindikatoren auf die kindliche Gesundheit vom Alter des Kindes bzw. Jugendlichen abhängen, wobei fünf Altersklassen gebildet werden (0-2 Jahre, 3-6 Jahre, 7-10 Jahre, 11-13 Jahre, 14-17 Jahre). Zum Beispiel zeigt sich, dass eine Skala zum Familienklima, die familiale Kohäsion misst, alters- und geschlechtsübergreifend erklärungskräftig ist: Kinder und Jugendliche werden bei einem besseren Familienklima durchgängig als signifikant gesünder eingestuft. Ein oder mehr Geschwisterteile beeinflussen dagegen lediglich die allgemeine Gesundheit von Jungen im Altersbereich 0-2 Jahre negativ, während sich für ältere Kindern und bei Mädchen keine Effekte zeigen.

Eine Besonderheit der Studie von Wu et al. (2010) ist, dass das familiale Sozialkapital im Rahmen von Strukturgleichungsmodellen analytisch als Mediator in eine Erklärungskette eingebettet wird. Exogene Variablen am Beginn dieser Kette sind das familiale Humankapital (elterliche Bildung) und das Familieneinkommen. Mediatoren bzw. intervenierende Variablen sind das familiale Sozialkapital (positive familiale Interaktion, soziale Kontrolle und Monitoring) und das „,community social capital“ (z. B. subjektiv als sicher empfundene Nachbarschaft, Anzahl der Freunde in der Nachbarschaft). Depression der Kinder stellt die Outcome-Variable dar. Im Rahmen von Pfadanalysen wird deutlich, dass das familiale Humankapital zum einen Depressivität direkt negativ beeinflusst und zum anderen indirekt negativ, da eine hohe Bildung der Eltern zu mehr familialem Sozialkapital führt, das seinerseits einen negativen Effekt auf die Depressivität der Kinder hat. Darüber hinaus führt auch ein höheres Sozialkapital in der Nachbarschaft zu einem stärker ausgeprägten familialen Sozialkapital und auf diese Weise zu einer geringeren Depressivität. Überraschenderweise haben gleichzeitig Familien mit höherem Familieneinkommen multivariat ein geringeres familiales Sozialkapital und sind auch etwas häufiger von Depressivität betroffen. 


\subsection{Elterliche soziale Netzwerke, familiales Sozialkapital und kindliche Gesundheit}

Die Literaturrecherche ergab vier Studien, die indirekte Effekte elterlicher sozialer Netzwerke in den Blick nehmen. Diese lassen sich wie folgt ordnen: Runyan et al. (1998) stützen ihre Analysen auf eine Stichprobe extremer Fälle, die durch spezielle Screening-Techniken gezogen wurde. Die Studien von Adams et al. (2002), Kennedy-Hendricks et al. (2015) sowie Kana'iaupuni et al. (2005) zeichnen sich zudem dadurch aus, dass hier eine Netzwerkmethodologie im engeren Sinne (hier: namensbasierte, egozentrierte Netzwerke) verwendet wird. Außerdem handelt es sich bei Adams et al. (2002) sowie Kana'iaupuni et al. (2005) um Studien, die nicht in westlichen Industrienationen durchgeführt wurden (Mali, Mexiko). In allen vier Fällen erscheint somit eine etwas ausführlichere Besprechung angemessen.

Runyan et al. (1998) verwenden eine Stichprobe (Longitudinal Studies on Child Abuse and Neglect, LONGSCAN), in der Kinder überrepräsentiert sind, die bereits seit ihrer Geburt besonderen gesundheitlichen Risiken ausgesetzt sind. Kriterien hierfür sind z. B. geringes Geburtsgewicht, alleinerziehendes Elternteil ohne familiale Unterstützung, geringes Alter der Mutter bei Geburt, Alkoholoder Drogenprobleme der Mutter, Misshandlung oder Wachstumsstörungen. Das kindliche Wohlbefinden wird über Indikatoren zu Entwicklungs- oder Verhaltensproblemen erfasst, z. B. Ängstlichkeit, Depression, körperliche Beschwerden, soziale Probleme, Konzentrations- und Schlafprobleme, regelverletzendes und aggressives Verhalten sowie motorische, adaptive, sprachliche und kognitive Fähigkeiten. Im Rahmen einer Fall-Kontroll-Studie werden Kinder, die auf dieser Basis als klinisch ,,auffällig“ klassifiziert werden, mit unauffälligen Kindern verglichen. Die Kinder sind im Mittel 4,5 Jahre alt.

Sozialkapital wird zum einen über die strukturelle Dimension nach Coleman (1990) (das Vorhandensein von zwei Eltern im Haushalt sowie die Geschwisteranzahl) gemessen. Zum anderen wird über sieben Globalindikatoren gemessen, inwieweit die primäre Betreuungsperson des Kindes über funktionale und emotionale Unterstützung aus ihrem sozialen Netzwerk verfügt (Beispiele: Ego kennt Personen, mit denen er über Probleme reden kann, erhält Einladungen, mit anderen auszugehen, erhält Liebe und Zuneigung, erhält Beratung zu wichtigen Lebensaspekten, wird bei eigener Krankheit unterstützt, kennt Personen, die sich darum kümmern, wie es ihm geht). Die so gemessene Form der sozialen Unterstützung wird einzeln betrachtet sowie mit anderen Sozialkapitalindikatoren (zwei Eltern im Haushalt, höchstens zwei Kinder, soziale Unterstützung aus der Nachbarschaft, regelmäßiger Kirchgang) zu einem Summenscore „Social Capital Index" verrechnet. Avancierte Netzwerkmaße werden nicht berichtet. 
Empirisch zeigt sich, dass die persönliche soziale Unterstützung der Eltern bei Kindern, die als auffällig klassifiziert werden, signifikant schwächer ausgeprägt ist als bei unauffälligen Kindern. Gleiches gilt für einen regelmäßigen Kirchgang und den Sozialkapitalindex insgesamt. Die strukturellen Indikatoren (zwei Eltern im Haushalt, nicht mehr als zwei Kinder) üben bivariat keinen signifikanten Einfluss aus. Der positive Effekt des Sozialkapitalindex auf die kindliche Entwicklung und Gesundheit bleibt auch in einem logistischen Regressionsmodell stabil, in dem für Bildung der Mutter, Familieneinkommen und mütterliche Depression kontrolliert wird.

Kennedy-Hendricks et al. (2015) zielen als einzige hier besprochene Studie auf Prozesse der sozialen Ansteckung ab. Die Stichprobe basiert auf eher benachteiligten Familien im Rahmen des sozialen Wohnungsbaus. Durch eine Erhebung namensbasierter egozentrierter sozialer Netzwerke der Eltern wird überprüft, inwiefern die Netzwerkpersonen (insbesondere in der Nachbarschaft) im Zusammenhang mit Gesundheitsaspekten positive Verhaltensmodelle für die Kinder darstellen, indem sie sich z. B. gesundheitsbewusst ernähren, physisch aktiv oder nicht übergewichtig sind. Empirisch zeigt sich, dass Kinder selbst sportlich aktiver und seltener übergewichtig sind, wenn sich in den Netzwerken der Eltern viele physisch aktive und nicht übergewichtige Personen befinden. Diese Ergebnisse unterstützen die unterstellten sozialen Lern- und Ansteckungsprozesse, auch wenn diese nicht direkt getestet werden.

Kana'iaupuni et al. (2005) zeigen mit Daten des Health und Migration Survey (HBS) für einige ausgewählte mexikanische Dörfer $(\mathrm{N}=620)$, dass sich das Ausmaß der emotionalen und finanziellen Unterstützung der Eltern durch ihre Netzwerkpersonen weniger mit der Anzahl der Blutsverwandten als mit der Anzahl der Personen (in unmittelbarer räumlicher Nähe) erhöht, die als erweiterte Verwandtschaft gelten. Hierzu zählen die oben bereits erwähnten Vertrauenspersonen und Paten der Kinder. Hoch interaktive soziale Netzwerke dieser Natur wirken sich zudem, vermittelt über emotionale und finanzielle Unterstützung, erwartungsgemäß positiv auf den allgemeinen Gesundheitszustand der Kinder aus insbesondere bei Familien, die über wenige materielle Ressourcen verfügen.

Adams et al. (2002) befragen $\mathrm{N}=1008$ Mütter in Mali aus zwei Volksstämmen (Bamanan, Fulbe). Durch einen Namensgenerator wird erfasst, wie groß spezifische soziale Teilnetzwerke sind, die für die Mutter materielle, praktische, kognitive und emotionale Unterstützung zur Verfügung stellen. Außerdem werden verschiedene Netzwerkcharakteristika relativ detailliert erhoben, z. B. die Geschlechts- und Alterskomposition, die räumliche Nähe der Netzwerkpersonen und der Beziehungsstatus zur Mutter (Verwandte, Freunde). Mithilfe einer Cox-Regression wird das Sterberisiko des Kindes in den ersten fünf Lebensjahren 
geschätzt. Die Sterbewahrscheinlichkeit des Kindes sinkt signifikant mit einer Zunahme der Netzwerkgröße sowie mit steigender Zahl von Personen, die praktische, kognitive und emotionale Unterstützung verfügbar machen. Dies gilt insbesondere für die Population der Fulbe, die besonders von Armut betroffen ist. Die verschiedenen strukturellen Netzwerkeigenschaften (z. B. Verwandtenanteil, Anteil der im Haushalt lebenden Netzwerkmitglieder) sind multivariat insignifikant.

\section{Familiales Sozialkapital, kindliche Gesundheit und soziale Ungleichheit}

In einem Zwischenkapitel werden nun das familiale Sozialkapital und die kindliche Gesundheit mit sozialer Ungleichheit in Verbindung gebracht, indem die referierten Studien aus diesem Blickwinkel kurz ausgewertet werden. Hierbei geht es um zwei Fragen:

1. Führt ein geringer sozioökonomischer Status der Eltern, vermittelt über eine geringe Ausstattung mit familialem Sozialkapital, zu einer schlechteren kindlichen Gesundheit? ${ }^{2}$

2. Besteht ein Interaktionseffekt zwischen dem sozioökonomischen Status und dem familialen Sozialkapital bei der Vorhersage der kindlichen Gesundheit? Wirkt sich also eine hohe Ausstattung mit Sozialkapital je nach sozialer Schicht anders auf die Gesundheit aus?

Zur ersten Frage ist zunächst festzustellen, dass insbesondere in den soziologischen Studien aus dem Bereich der familialen Sozialkapitalforschung multivariate Modelle berechnet werden, in denen sowohl die Sozialkapital-Indikatoren als auch Merkmale zur sozioökonomischen Lage (Bildung, Einkommen und Erwerbsstatus der Eltern) vertreten sind. Generell verdeutlichen die Befunde, dass das familiale Sozialkapital seine eigenständige Erklärungskraft auch bei Kontrolle der sozioökonomischen Indikatoren behält. Im Hinblick auf die erste zu beantwortende Frage bedeutet dies, dass das Sozialkapital nicht bloß eine Funktion der sozioökonomischen Situation ist, sondern eigenständige Effekte hat.

\footnotetext{
${ }^{2}$ Für eine ausführliche theoretische Diskussion des Zusammenhangs zwischen sozioökonomischen Status und Sozialkapital siehe Hartung (2013, S. 139-175).
} 
Gleichzeitig zeigen diejenigen Studien, die entweder hierarchische Regressionsmodelle oder - wie Wu et al. (2010) - Strukturgleichungsmodelle verwenden, dass das familiale Sozialkapital sowohl von anderen Sozialkapital-Subdimensionen (Schule, Nachbarschaft) als auch vom sozioökonomischen Status der Familie abhängig ist. Wu et al. (2010) differenzieren diese Abhängigkeit dahin gehend, dass vor allem Familien mit hoher elterlicher Bildung über mehr Sozialkapital (hier vor allem familiale Kohäsion) verfügen. Diese Befunde bestätigen die in Frage 1 formulierte Vermittlungshypothese. ${ }^{3}$

Eine allgemeine soziologische Erklärung besteht darin, dass sozio-ökonomische Benachteiligung nicht nur mit materiellen Einschränkungen einhergeht, sondern auch zu geringeren Partizipationschancen und schwächerer sozialer Integration führt: Sozial benachteiligte Menschen erweitern ihre sozialen Netzwerke in der Regel nicht, sondern ziehen sich auf verwandtschaftliche und enge Freundeskreise zurück, die jedoch häufig genauso ressourcenschwach sind wie die Betroffenen selbst (vgl. Hartung 2013, S. 73 ff). Wu et al. (2010) postulieren zudem, dass die sozioökonomische Lage der Familie auch über die Wohnstandortwahl entscheidet und damit gleichzeitig über die Ressourcen, die über nachbarschaftliche Beziehungen mobilisiert werden können. Nach Coleman (1990) ist darüber hinaus zu erwarten, dass wohlhabende Eltern besonders viel Ressourcen in die Qualität der Eltern-Kind-Beziehung investieren, da die Transmission der elterlichen Bildung und des finanziellen Wohlstands auf die Kinder intensive Eltern-Kind-Interaktionen voraussetzt. Die Befundlage stimmt ferner mit der Angleichungsthese von West (1997) überein. Hiernach werden in der frühen Kindheit Unterschiede in der Gesundheit relativ stark von der sozioökonomischen Position der Familie beeinflusst, die sich - nach den hier diskutierten Befunden - auch in einer spezifischen Ausstattung mit Sozialkapital niederschlägt. Im frühen Jugendalter verschwinden gesundheitsspezifische Unterschiede zwischen den verschiedenen sozialen Schichten dagegen weitgehend, da von Schule, Peergroup und Jugendkultur nivellierende Einflüsse ausgehen.

Zur zweiten Frage ist auf die Studie von Klocke (2004) einzugehen, die jedoch mit einem Altersbereich von 11-15 Jahren eher auf Jugendliche fokussiert. Hier wird ein Interaktionseffekt zwischen materiellen Ressourcen und Sozialkapital bei der Vorhersage der Gesundheit getestet. Die Befunde verdeutlichen, dass sich das gesundheitsrelevante Verhalten (hier: Rauchen) in allen sozialen

\footnotetext{
${ }^{3} \mathrm{Nach}$ Wu et al. (2010) ist dabei jedoch entscheidend, wie der sozioökonomische Status der Eltern operationalisiert wird. So erbringt die Studie den überraschenden Befund, dass die finanziellen Ressourcen der Eltern zu einer größeren Depressivität des Kindes führen. Erklärt wird dies dadurch, dass die Finanzressourcen ein indirekter Indikator für den Arbeitsumfang der Eltern sind, der die Zeit für gemeinsame Eltern-Kind-Interaktionen reduziert.
} 
Ungleichheitslagen mit steigender familialer Kohäsion und Unterstützung des Kindes durch die Eltern verbessert. Auch wenn geringe materielle Ressourcen oft mit niedrigem Sozialkapital einhergehen, so können hiernach Kinder und Jugendliche in allen Soziallagen von einer guten Sozialkapital-Ausstattung profitieren.

Auch wenn sich somit für dieses Beispiel aus Deutschland kein Interaktionseffekt zwischen sozioökonomischen Status und Sozialkapital bei der Vorhersage der Gesundheit zeigt (und Forschungsfrage 2 hier somit verneint werden muss), so ergeben die Studien zu nicht-westlichen Industrienationen (Adams et al. 2002; Kana'iaupuni et al. 2005) ein anderes Bild: Unter der Bedingung von stärkerer materieller Deprivation (Mexiko) bzw. extremer Armut (Mali) wird das Sozialkapital zu einem kompensierenden bzw. existenziellen Faktor für die Gesundheit bzw. das Überleben der Kinder. Auch innerhalb der beiden hier besprochenen Länder zeigt sich, dass die Kinder besonders armer Familien am meisten von sozialer Unterstützung profitieren, die den Eltern zur Verfügung steht.

\section{Abschließende Diskussion des Forschungsstands}

Zunächst ist allgemein festzustellen, dass die Netzwerkperspektive sowie der Ansatz des familialen Sozialkapitals bei der Erklärung kindlicher Gesundheit ein großes Potenzial haben. In fast allen Fällen zeigen sich die erwarteten positiven Korrelationen mit den Gesundheitsindikatoren.

Dennoch sind einige Aspekte kritisch zu diskutieren. Insbesondere die soziologischen Sozialkapitalstudien gehen explizit oder implizit davon aus, dass die Eltern die wesentlichen Netzwerkpersonen sind, durch die das Kind Zugriff auf wichtige Ressourcen wie Aufmerksamkeit oder Unterstützung erhält. Darüber hinaus werden gelegentlich noch Geschwister berücksichtigt. Weitere mögliche Mitglieder des Familiennetzwerks, wie z. B. Großeltern, Onkel oder Tanten und Paten, finden dagegen - im Gegensatz zum ausgereifteren Konvoy-Modell der Autorengruppe um Mary J. Levitt - keine Beachtung. ${ }^{4}$ Insgesamt sind Studien,

\footnotetext{
${ }^{4}$ Ein weiterer Vorschlag, um breitere Familienkonfigurationen zu erfassen, stammt von Widmer (2006). Bei der hier entwickelten „family network method“ handelt es sich im Wesentlichen um Namensgeneratoren, bei denen Ego gebeten wird, signifikante Familienmitglieder zu nennen, die zurzeit oder im letzten Jahr eine wichtige Rolle in seinem Leben gespielt haben. Dabei kann es sich sowohl um geliebte und respektierte Personen als auch um Personen handeln, mit denen Konflikte bestehen. Eine Befragung auf Basis einer Studentenpopulation, die auf dieser Methode aufbaut, führt zu verschiedenen Typen von familialen sozialen Netzwerken, die neben Blutsverwandten auch Stiefeltern und Freunde umfassen, die subjektiv als signifikante Familienmitglieder betrachtet werden (z. B. Taufpaten).
} 
die „echte“ Netzwerkanalysen (z. B. egozentrierte Studien) durchführen, im vorliegenden Kontext selten.

Bei einer Kausalinterpretation der referierten Befunde ist in einigen Fällen auf Alternativerklärungen hinzuweisen. So wird z. B. von Martin (2008) ausgeführt, dass der Zusammenhang zwischen dem Gewicht der Eltern und ihrer Kinder zu einem großen Anteil auf genetische Faktoren zurückzuführen ist - zum einen dadurch, dass sich die physiologischen Voraussetzungen für das Körpergewicht vererben und zum anderen auch dadurch, dass Prädispositionen für bestimmte Lebensstilmerkmale (z. B. physische Aktivität) zum Teil genetisch bedingt sind. Wird diese genetische Störgröße im Rahmen einer Zwillingsstudie ${ }^{5}$ statistisch kontrolliert, zeigt sich, dass sozial verursachte Lebensstilfaktoren sowohl an Erklärungskraft verlieren (Mediation) als auch an Bedeutung gewinnen können, d. h. durch genetische Faktoren bivariat verdeckt werden (Suppression). In den hier besprochenen Studien wird in der Regel nicht für genetische Faktoren kontrolliert, wodurch sich zum Teil Interpretationsprobleme ergeben.

Schultz et al. (2009) gehen ferner der Frage nach, ob die kindliche Gesundheit einen Effekt auf das Sozialkapital der Eltern hat. Ließen sich Hinweise auf eine derartige umgekehrte Kausalität finden, würde dies die referierten Befunde in ihrer bisherigen Lesart zum Teil in Zweifel stellen. Empirisch zeigt sich allerdings in einer Studie von Eltern, die ab Geburt in einem Dreijahreszeitraum beobachtet werden, dass mehr oder weniger ernsthafte gesundheitliche Probleme des Babys (z. B. geringes Geburtsgewicht, Behinderungen) im Sinne eines exogenen Schocks, der durch die elterlichen Verhaltensweisen nicht selbst herbeigeführt wurde, keinen Effekt auf verschiedene Sozialkapitalindikatoren (z. B. Häufigkeit des Besuchs bei Verwandten, Kirchgangshäufigkeit und Aktivität in Organisationen) haben.

Im Hinblick auf die theoretischen Mechanismen ist festzustellen, dass ein expliziter Test bisher am ehesten für den Mechanismus der sozialen Unterstützung erfolgt ist. Während das Unterstützungspotenzial aus dem Netzwerk in einer Reihe von Studien durch spezifische Indikatoren facettenreich erhoben wird, fehlen ausgereifte Messinstrumente und Forschungsdesigns für die Mechanismen der sozialen Kontrolle oder der sozialen Ansteckung weitgehend. Hier besteht weiterhin großer Forschungsbedarf.

Ein weiterer interessanter Aspekt betrifft schließlich das Wechselverhältnis zwischen informeller sozialer Unterstützung in sozialen Netzwerken und

\footnotetext{
${ }^{5}$ Mehrgruppenvergleiche in Strukturgleichungsmodellen mit monozygotischen und dizygotischen Zwillingen.
} 
professioneller Hilfe bei gesundheitlichen Problemen. So zeigen Martinez und Lau (2011) in einer US-amerikanischen Studie, dass Eltern, deren Kinder mentale Gesundheitsprobleme aufweisen, bei hoher wahrgenommener Unterstützung aus dem sozialen Netzwerk weniger dazu tendieren, professionelle Hilfsangebote in Anspruch zu nehmen. Dies ist teilweise darauf zurückzuführen, dass sich der Gesundheitszustand der Kinder bei hohem Unterstützungspotenzial tatsächlich über die Zeit verbessert.

\section{Leseempfehlungen}

Alvarez, E. C., Kawachi, I., \& Romani, J. R. (2017). Family social capital and health: A systematic review and redirection. Sociology of Health \& Illness, 39(1), S. 5-29. Altersübergreifender Überblick zur Forschung im Bereich des familialen Sozialkapitals

Morrow, V. (1999). Conceptualising social capital in relation to the wellbeing of children and young people: A critical review. Sociological Review, 47 (4), S. 744-765. Kritische Diskussion des Sozialkapital-Ansatzes

\section{Datensätze}

- Studie zur Gesundheit von Jugendlichen in Deutschland (KiGGS) Im Rahmen der vom Robert Koch-Institut durchgeführten KiGGS-Studie wurden 2003-2006 erstmals umfassende und bundesweit repräsentative Gesundheitsdaten für Kinder und Jugendliche erhoben. Seit 2009 wird KiGGS als Langzeitstudie fortgeführt. Mithilfe der Daten können sowohl die aktuelle gesundheitilche Lage von Kindern und Jugendlichen unter 18 Jahren als auch - aufgrund der Panelstruktur der Daten - zeitliche Entwicklungstrends und Veränderungen im Lebensverlauf analysiert werden. Familiales Sozialkapital lässt sich über verschiedene Globalindikatoren abbilden die z. B. familiale Kohäsion oder elterliche soziale Kontrolle messen.

Zugang über www.kiggs-studie.de

- Health Behaviour in School-Aged Children - WHO Collaborative Cross-National Survey (HBSC)

Die alle vier Jahre stattfindende Befragung HSBC wurde 1982 initiiert und wird derzeit in 48 Ländern (Europa und Nordamerika) durchgeführt. Das Ziel der Studie ist die Sammlung landesweit repräsentativer 
Daten über die Gesundheit, das familiäre und soziale Umfeld sowie das gesunheitsrelevante Verhalten von Jungen und Mädchen des 5. bis 9. Schuljahres, die in der Regel zwischen 11 und 15 Jahre alt sind. Familiales Sozialkapital wird über einige Globalindikatoren wie z. B. emotionale oder instrumentelle Unterstützung durch die Eltern operationalisiert.

Zugang über www.hbsc.org (international) bzw. hbsc-germany.de (Deutschland)

\section{Literatur}

Adams, A. M., Madhavan, S., \& Simon, D. (2002). Women's social networks an child survival in Mali. Social Science and Medicine, 54, 165-178.

Alvarez, E. C., Kawachi, I., \& Romani, J. R. (2017). Family social capital and health: A systematic review and redirection. Sociology of Health \& Illness, 39, 5-29.

Bala-Brusilow, C. (2010). A Study of the Associations between Childhood Obesity and three Forms of Social Capital. Wayne State University.

Bandura, A. (1977). Social Learning Theory. Englewood Cliffs: Prentice Hall.

Berntsson, L., Köhler, L., \& Vuille, J.-C. (2007). Health, economy and social capital in nordic children and their families: A comparison between 1984 and 1996. Child: Care. Health \& Development, 32, 441-451.

Boosman, K., van der Meulen, M., van Geert, P., \& Jackson, S. (2002). Measuring young children's perceptions of support, control, and maintenance in their own social networks. Social Development, 11(3), 386-408.

Bost, K. K., Vaughn, B. E., Boston, A. L., Kazura, K. L., \& O’Neal, C. (2004). Social support networks of African-American children attending head start: A longitudinal investigation of structural and supportive network characteristics. Social Development, 13(3), 393-412.

Bowlby, J. (1975). Bindung. Frankfurt: Fischer.

Bronfenbrenner, U. (1981). Die Ökologie der menschlichen Entwicklung. Natürliche und geplante Experimente. Stuttgart: Klett-Cotta.

Cochran, M. M., \& Brassard, J. A. (1979). Child development and personal social networks. Child Development, 50(3), 601-616.

Coleman, J. S. (1990). Foundations of the Social Theory. Cambridge: Harvard University Press.

Dilworth-Anderson, P., Burton, L. M., \& Klein, D. M. (2005). Contemporary and emerging theories in studying families. In V. L. Bengtson, A. C. Acock, K. R. Allen, P. DilworthAnderson, \& D. M. Klein (Hrsg.), Sourcebook of Family Theory and Research (S. 35-58). Thousand Oaks: Sage. 
Ellsäßer, G., \& Lüdecke, K. (2015). Einschuluntersuchung 2015. Zossen: Landesamt für Arbeitsschutz, Verbraucherschutz und Gesundheit des Landes Brandenburg.

Erhart, M., \& Ravens-Sieberer, U. (2008). Die Rolle struktureller Aspekte von Familie, innerfamiliärer Kommunikation und Unterstützung für die Gesundheit im Kindes- und Jugendalter. In M. Richter, K. Hurrelmann, A. Klocke, W. Melzer, \& U. Ravens-Sieberer (Hrsg.), Gesundheit, Ungleichheit und jugendliche Lebenswelten. Ergebnisse der zweiten internationalen Vergleichsstudie im Auftrag der Weltgesundheitsorganisation WHO (S. 190-230). Weinheim: Juventa.

Eriksson, U., Hochwälder, J., Carlsund, A., \& Sellström, E. (2012). Health outcomes among Swedish children: The role of social capital in the family, school and neighbourhood. Acta Paediatrica, 101, 513-517.

Geyer, S., \& Peter, R. (1998). Unfallbedingte Krankenhausaufnahme von Kindern und Jugendlichen in Abhängigkeit von ihrem sozialen Status - Befunde mit Daten einer nordrhein-westfälischen AOK. Gesundheitswesen, 60, 493-499.

Hartung, S. (2013). Sozialkapital und gesundheitliche Ungleichheit. Analyse des elterlichen Sozialkapitals in der schulischen Gesundheitsförderung. Wiesbaden: Springer VS.

Hradil, S. (1987). Sozialstrukturanalyse in einer fortgeschrittenen Gesellschaft. Opladen: Leske+Budrich.

Kahn, R. L., \& Antonucci, T. C. (1980). Convoys over the life course: Attachment, roles, and social support. In P. B. Baltes \& O. G. Brim (Hrsg.), Life-Span Development and Behaivor (S. 253-586). New York: Academic.

Kana'iaupuni, M. S., Donato, K. M., Thompson-Colón, T., \& Steinback, M. (2005). Counting on kin: Social networks, social support, and child health status. Social Forces, 83, 1137-1164.

Kennedy-Hendricks, A., Schwartz, H., Johnson Thornton, R., Griffin, B. A., Green, H. D., Jr., Kennedy, D. P., Burkhauser, S., \& Pollack, C. E. (2015). Intergenerational social networks and health behaviors among children living in public housing. American Journal of Public Health, 105, 2291-2297.

Klocke, A. (2004). Soziales Kapital als Ressource für Gesundheit im Jugendalter. In M. Jungbauer-Gans \& P. Kriwy (Hrsg.), Soziale Benachteiligung und Gesundheit bei Kindern und Jugendlichen (S. 85-96). Wiesbaden: Springer VS.

Klocke, A., \& Lipsmeier, G. (2008). Soziale Determinanten der Gesundheit im Kindesund Jugendalter: eine Mehrebenenanalyse. In M. Richter, K. Hurrelmann, A. Klocke, W. Melzer, \& U. Ravens-Sieberer (Hrsg.), Gesundheit, Ungleichheit und jugendliche Lebenswelten (S. 231-254). München: Juventa.

Lampert, T., \& Richter, M. (2009). Gesundheitliche Ungleichheit bei Kindern und Jugendlichen. In M. Richter \& K. Hurrelmann (Hrsg.), Gesundheitliche Ungleichheit. Grundlagen, Probleme, Perspektiven (S. 207-228). Wiesbaden: Springer VS.

Lampert, T., \& Schenk, L. (2004). Gesundheitliche Konsequenzen des Aufwachsens in Armut und sozialer Benachteiligung. In M. Jungbauer-Gans \& P. Kriwy (Hrsg.), Soziale Benachteiligung und Gesundheit bei Kindern und Jugendlichen (S. 57-83). Wiesbaden: Springer VS.

Lau, M., \& Li, W. (2011). The extent of family and school social capital promoting positive subjective well-being among primary school children in Shenzhen, China. Children and Youth Services Review, 33, 1573-1582. 
Levitt, M. J., Guacci-Franco, N., \& Levitt, J. L. (1993). Convoys of social support in childhood and early adolescence: Structure and function. Developmental Psychology, 29(5), 811-818.

Levitt, M. J., Levitt, J. L., Bustos, G. L., Crooks, N. A., Santos, J. D., Telan, P., Hodgetts, J., \& Milevsky, A. (2005). Patterns of social support in the middle childhood to early adolescent transition: Implications for adjustment. Social Development, 14(3), 398-420.

Martin, M. A. (2008). The intergenerational correlation in weight: How genetic resemblance reveals the social role of families. American Journal of Sociology, 114(Supplement), 67-105.

Martinez, J. I., \& Lau, A. S. (2011). Do social networks push families toward or away from youth mental health services?: A national study of families in child welfare. Journal of Emotional and Behavioral Disorders, 19, 169-181.

Mielck, A. (1998). Armut und Gesundheit bei Kindern und Jugendlichen: Ergebnisse der sozial-epidemiologischen Forschung in Deutschland. In A. Klocke \& K. Hurrelmann (Hrsg.), Kinder und Jugendliche in Armut: Umfang, Auswirkungen und Konsequenzen (S. 225-249). Wiesbaden: Westdeutscher Verlag.

Morgan, A., \& Haglund, B. J. A. (2009). Social capital does matter for adolescent health: Evidence from the English HBSC Study. Health Promotion International, 24, 363-372.

Morrow, V. (1999). Conceptualising social capital in relation to the well-being of children and young people: A critical review. Sociological Review, 47, 744-765.

Nestmann, F., \& Wehner, K. (2008). Soziale Netzwerke von Kindern und Jugendlichen. In F. Nestmann, J. Günther, S. Stiehler, K. Wehner, \& J. Werner (Hrsg.), Kindernetzwerke. Soziale Beziehungen und soziale Unterstützung in Familie, Pflegefamilie und Heim (S. 11-40). Tübingen: dgvt-Verlag.

Rattay, P., Lampert, T., Neuhauser, H., \& Ellert, U. (2012). Bedeutung der familialen Lebenswelt für die Gesundheit von Kindern und Jugendlichen. Ergebnisse des Kinderund Jugendgesundheitssurveys (KiGGS). Zeitchrift für Erziehungswissenschaft, 15, 145-170.

Richter, M., Hurrelmann, K., Klocke, A., Melzer, W., \& Ravens-Sieberer, U. (Hrsg.). (2008). Gesundheit, Ungleichheit und jugendliche Lebenswelten: Ergebnisse der zweiten internationalen Vergleichsstudie der Weltgesundheitsorganisation WHO. Weinheim: Juventa.

Runyan, D. K., Hunter, W. M., Socolar, R. R. S., Amaya-Jackson, L., English, D., Landsverk, J., Dubowitz, H., Browne, D. H., Bangdiwala, S. I., \& Mathew, R. M. (1998). Children who prosper in unfavorable environments: The relationship to social capital. Pediatrics, 101, 12-18.

Schultz, J., Corman, H., \& Reichman, N. E. (2009). Effects of child health on parents“ social capital. Social Science and Medicine, 69, 76-84.

Tiber Egle, U., Hardt, J., \& Ralf, N. (2002). Früher Streß und Langzeitfolgen für die Gesundheit - wissenschaftlicher Erkenntnisstand und Forschungsdesiderate. Zeitschrift für Psychosomatische Medizin und Psychotherapie, 4, 411-434.

Vaux, A. (1988). Social Support: Theory, Research and Intervention. New York: Praeger.

West, P. (1997). Health inequalities in the early years: Is there equalisation in youth? Social Science and Medicine, 27, 291-296.

Widmer, E. D. (2006). Who are my family members? Bridging and binding social capital in family configurations. Journal of Social and Personal Relationships, 23, 979-998. 
Wippermann, C. (2009). Lebensstile und Milieus: Einflüsse auf die Gesundheit. In V. Schumpelick \& B. Vogel (Hrsg.), Volkskrankheiten: Gesundheitliche Herausforderungen in der Wohlstandsgesellschaft (S. 143-156). Freiburg i.Br.: Herder.

Wu, Q., Xie, B., Chou, C.-P., Palmer, P. H., Gallaher, P. E., \& Jonson, A. C. (2010). Understanding the effect of social capital on the depression of urban Chinese adolescents: An integrative framework. American Journal of Community Psychology, 45, $1-16$.

Open Access Dieses Kapitel wird unter der Creative Commons Namensnennung 4.0 International Lizenz (http://creativecommons.org/licenses/by/4.0/deed.de) veröffentlicht, welche die Nutzung, Vervielfältigung, Bearbeitung, Verbreitung und Wiedergabe in jeglichem Medium und Format erlaubt, sofern Sie den/die ursprünglichen Autor(en) und die Quelle ordnungsgemäß nennen, einen Link zur Creative Commons Lizenz beifügen und angeben, ob Änderungen vorgenommen wurden.

Die in diesem Kapitel enthaltenen Bilder und sonstiges Drittmaterial unterliegen ebenfalls der genannten Creative Commons Lizenz, sofern sich aus der Abbildungslegende nichts anderes ergibt. Sofern das betreffende Material nicht unter der genannten Creative Commons Lizenz steht und die betreffende Handlung nicht nach gesetzlichen Vorschriften erlaubt ist, ist für die oben aufgeführten Weiterverwendungen des Materials die Einwilligung des jeweiligen Rechteinhabers einzuholen.

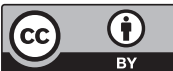

